hep-th/9903210; LPTENS-99-07; DAMTP-1999-14

\title{
Curvature terms in D-brane actions and their M-theory origin
}

\author{
Constantin P. Bachast, Pascal Bain2 \\ Laboratoire de Physique Théorique de l'École Normale Supérieure $\mathrm{B}$ \\ 24 Rue Lhomond, 75231 Paris Cedex, France \\ and \\ Michael B. Green团 \\ Department of Applied Mathematics and Theoretical Physics \\ Silver Street, Cambridge CB3 9EW, UK
}

\begin{abstract}
We derive the complete (curvature ${ }^{2}$ terms of effective D-brane actions, for arbitrary ambient geometries and world-volume embeddings, at lowest order (disk-level) in the string-loop expansion. These terms reproduce the $o\left(\alpha^{\prime 2}\right)$ corrections to string scattering amplitudes, and are consistent with duality conjectures. In the particular case of the D3brane with trivial normal bundle, considerations of $S L(2, \mathbb{Z})$ invariance lead to a complete sum of D-instanton corrections for both the parity-conserving and the parity-violating parts of the effective action. These corrections are required for the cancellation of the modular anomalies of massless modes, and are consistent with the absence of chiral anomalies in the intersection domain of pairs of D-branes. We also show that the parity-conserving part of the non-perturbative $R^{2}$ action follows from a one-loop quantum calculation in the six-dimensional world-volume of the M5-brane compactified on a two-torus.
\end{abstract}

$2 / 99$

1 email: bachas@physique.ens.fr

2 email: bain@physique.ens.fr

3 Unité mixte du CNRS, UMR8549 .

4 email: M.B.Green@damtp.cam.ac.uk 


\section{Introduction}

The dynamics of $p$-branes is well-approximated by effective world-volume $(p+1)$ dimensional field theories of various kinds when $p<5$. In the case of Dp-branes the effective world-volume actions consist of the sum of a Wess-Zumino (WZ) part that is parity-violating, and of a parity-conserving part whose first term is the Dirac-Born-Infeld (DBI) action (see [1,2] for reviews).

The WZ term describes the coupling of a Dp-brane to the bulk Ramond-Ramond $(\mathcal{R} \otimes \mathcal{R})$ fields and can be motivated in a variety of manners [3, 4, 4, 6, 6, 7,8,9] . It has the structure of an anomaly cancelling term and can indeed be determined by requiring the consistent cancellation of the chiral gauge and gravitational anomalies that arise when pairs of D-branes intersect in certain configurations [8,9]. Such terms have a simple topological description which results in the expression for the $(p+1)$-form WZ term

$$
\int_{M^{(p+1)}} \mathcal{L}_{W Z}^{(p) d i s k}=T_{(p)} \int_{M^{(p+1)}} C \wedge \operatorname{tr}_{N}\left(e^{2 \pi \alpha^{\prime} F}\right) \wedge\left(\frac{\hat{\mathcal{A}}\left(4 \pi^{2} \alpha^{\prime} R_{T}\right)}{\hat{\mathcal{A}}\left(4 \pi^{2} \alpha^{\prime} R_{N}\right)}\right)^{1 / 2},
$$

where $T_{(p)}$ is the tension of the Dp-brane and the trace is in the fundamental representation of the world-volume gauge group, $U(N)$. The expression $\hat{\mathcal{A}}$ is the Dirac 'roof' genus and its square root has the expansion in even powers of the curvature two-form,

$$
\sqrt{\hat{\mathcal{A}}(R)}=1-\frac{1}{48} p_{1}(R)+\frac{1}{2560} p_{1}^{2}(R)-\frac{1}{2880} p_{2}(R)+\ldots,
$$

where $p_{1}$ and $p_{2}$ are the first two Pontryagin classes. The components of the curvature with tangent-space indices are denoted by $R_{T}$ in (1.1) while $R_{N}$ denotes the components in the normal bundle (the definitions of our notation are made precise in the appendices). The $\mathcal{R} \otimes \mathcal{R}(p+1)$-form potentials pulled back to the Dp-brane are contained in $C=$ $C^{(0)}+C^{(1)} \ldots+C^{(9)}$, where the odd forms contribute in the IIA theory and the even ones in the IIB theory, The structure of (1.1) may also be determined by considering the scattering of gravitons on a world-sheet with disk topology [10,11, 12], which we will refer to as the 'tree-level' contribution. As usual, since these terms are responsible for the cancellation of anomalies they receive no perturbative corrections.

The DBI action for a single Dp-brane [13,14] encapsulates the exact dependence on the field strength of the Born-Infeld vector potential for constant field strengths, and can be fixed by considerations of T-duality and Lorentz covariance [15]

$$
\int_{M^{(p+1)}} \mathcal{L}_{D B I}^{(p)}=T_{(p)} \int_{M^{(p+1)}} e^{-\phi} \sqrt{\operatorname{det}\left(\left(G_{\mu \nu}+B_{\mu \nu}\right) \partial_{\alpha} Y^{\mu} \partial_{\beta} Y^{\nu}+2 \pi \alpha^{\prime} F_{\alpha \beta}\right)} .
$$


Here and in the following, we will use Greek indices from the middle of the alphabet $(\mu, \nu, \ldots)$ for the space-time coordinates, Greek letters from the beginning of the alphabet $(\alpha, \beta \ldots)$ for the world-volume coordinates, and Latin letters $(a, b, \ldots)$ for the normal bundle (see appendix A for more details). In contrast to the WZ action, the expression (1.3) does not include gravitational-curvature effects induced by the ambient geometry and/or by non-trivial world-volume embeddings. Such effects will generically require the addition of terms that again involve powers of the Riemann curvature with nontrivial values in both the tangent bundle and the normal bundle. In section 2 we will determine the structure of these terms by considering the scattering of gravitons and of ripples (open strings) in the world-volume of the D-brane. A residual ambiguity will be fixed by invoking heterotic/type II duality in six dimensions. Our analysis in section 2 will be restricted to the lowest-order terms, which arise from world-sheets with the topology of a disk and which are quadratic in the curvature expansion. For simplicity we will also assume constant dilaton and $\mathcal{R} \otimes \mathcal{R}$ backgrounds, as well as vanishing $B_{\mu \nu}$ and $F_{\alpha \beta}$ fields, but we allow for arbitrary ambient geometries and world-volume embeddings.

In section 3 we will consider the particular case of D3-branes. We will explain why the curvature-squared terms receive non-perturbative D-instanton corrections that are necessary in order to ensure that the type IIB theory maintains the requisite $S L(2, \mathbb{Z})$ invariance [16, 177 in the presence of D3-branes. The terms at issue are of the form $f(\tau, \bar{\tau}) R^{2}$, where $R$ denotes the components of the Riemann curvature and the function $f(\tau, \bar{\tau})$ has specified properties under modular transformations of the complex scalar field of the IIB theory,

$$
\tau \equiv \tau_{1}+i \tau_{2}=\frac{1}{\lambda_{s}^{B}}\left[C^{(0)}+i e^{-\phi}\right]
$$

where $C^{(0)}$ is the $\mathcal{R} \otimes \mathcal{R}$ scalar and $\phi$ is the type IIB dilaton. In the special case of a trivial normal bundle we will see that the complete expression for the $R^{2}$ terms in the D3-brane action will be given by,

$$
\int_{M^{(4)}} \mathcal{L}_{R^{2}}^{(3)}=\frac{1}{16 \pi^{2}} \int_{M^{(4)}} \operatorname{Re}\left[\log \eta(\tau) \operatorname{tr}\left(\mathrm{R} \wedge^{*} \mathrm{R}-i \mathrm{R} \wedge \mathrm{R}\right)\right],
$$

where $\eta(\tau)$ is the Dedekind function, $\mathrm{R} \equiv \frac{1}{2} R_{\alpha \beta} d \zeta^{\alpha} \wedge d \zeta^{\beta}$ is the $S O(1,3)$-valued curvature two-form, and ${ }^{*} \mathrm{R} \equiv \frac{1}{4} \sqrt{g} \epsilon_{\alpha \beta}{ }^{\gamma \delta} R_{\gamma \delta} d \zeta^{\alpha} \wedge d \zeta^{\beta}$ is its Hodge dual. The expression (1.5) has

5 The D-brane tensions and the gravitational coupling are given by $2 \kappa_{(10)}^{2}=\lambda_{s}^{2} \alpha^{\prime 4}(2 \pi)^{7}$ and $\kappa_{(10)}^{2} T_{(p)}^{2}=\pi\left(4 \pi^{2} \alpha^{\prime}\right)^{3-p}$. The symbols $\lambda_{s}^{A}$ and $\lambda_{s}^{B}$ will be used to distinguish the string couplings in the IIA and IIB theories, respectively, and the dilaton $\phi$ has zero vacuum expectation value. 
a small-coupling expansion $\left(\tau_{2} \rightarrow \infty\right)$ that contains the 'tree-level' terms that arise from the disk calculations in section 2 together with an infinite series of D-instanton corrections.

One argument for the form (1.5) of the D3-brane action, based on type IIA/F-theory duality [18], will be described in the first part of section 3. The F-theory background contains $24(p, q)$ seven-branes, each making an equal contribution to the $R^{2}$ terms of the effective low-energy action. This universal piece of the seven-brane action is related by T-duality to the $R^{2}$ terms of the D3-brane action. On the type-IIA side, on the other hand, these same terms come from a one-loop amplitude which was computed in [19,20]. Comparing the two sides leads to expression (1.5) .

A different argument for the structure of (1.5) follows by careful consideration of its properties under $S L(2, \mathbb{Z})$ transformations. We will see in the second part of section 3 that the conjectured action transforms under $S L(2, \mathbb{Z})$ transformations in precisely the appropriate manner to cancel the $\tau$-dependent anomalous transformation of the determinant of the massless world-volume fields. This anomaly results from the chiral couplings of the world-volume fields to the $S L(2, \mathbb{R})$ Noether current. A $\tau$-independent modular anomaly remains, but it is the integral of a topological density which integrates to an integer multiple of $2 \pi$ in consistent backgrounds

The emphasis in section 4 is on the relationships between the nonperturbative curvature terms in the D3-brane of the IIB theory and properties of the M5-brane compactified on $T^{2}$. Besides specializing to the case of a trivial normal bundle, we also only consider the parity conserving terms. We will make use of the various dualities that relate M-theory on $T^{2}$ to the type IIA and IIB theories compactified on $S^{1}$ [21,22] to relate the D3-brane to higher-dimensional branes. T-duality on the circle relates the IIB theory to the IIA theory, and converts an unwrapped D3-brane into a wrapped D4-brane. The instanton terms in the D3-brane are now interpreted as the world-lines of D-particles which form bound states with the D4-brane [1]. These are the expected threshold bound states of a quantum mechanical system with $\mathcal{N}=(4,4)$ supersymmetry. As we will see, the exact expression for the D-instanton measure that is extracted from the function $f(\tau, \bar{\tau})$ is consistent with the fact that the Witten index for this D-particle-D4-brane is one. The D4-brane in turn may be interpreted as the M5-brane wrapped around the circular eleventh dimension of M-theory. The D-particle bound states are then simply the Kaluza-Klein excitations in the double dimensional reduction of the M5-brane. In this picture the $R^{2}$ terms can be 
deduced by a calculation of a one-loop effect in the world-volume of the compactified M5brane which will be described in section 4 . This is analogous to the manner in which the $R^{4}$ terms of the bulk theory were deduced in [23].

Section 5 summarizes our conclusions, and outlines how our arguments could be extended in several directions. We discuss in particular why non-perturbative $R^{4}$ terms are expected in the world-volume action of a seven-brane, and how the analysis of sections 3 and 4 could be extended to account for non-trivial normal bundles. Finally we comment on the relevance of our results in the context of the AdS/CFT correspondence.

\section{Disk-level curvature terms}

In this section we will extract the $R^{2}$ terms of the classical D-brane action from disk-level scattering amplitudes, combined with a duality argument and the requirement of reparametrization invariance. We begin by reviewing the calculation [24,25] of the disk diagram for (a) the elastic scattering of a graviton off a D-brane, (b) the absorption of a graviton whose energy is carried away by two ripples (represented by open strings) in the world-volume of the D-brane, and (c) the elastic scattering of two such worldvolume ripples. These processes can all be conveniently expressed in terms of the standard amplitude for the scattering of four open-string gauge bosons 26]

$$
A\left(\zeta_{1}, k_{1} ; \zeta_{2}, k_{2} ; \zeta_{3}, k_{3} ; \zeta_{4}, k_{4}\right)=\mathcal{N} K(1,2,3,4) \frac{\Gamma\left(2 \alpha^{\prime} k_{1} \cdot k_{2}\right) \Gamma\left(2 \alpha^{\prime} k_{1} \cdot k_{4}\right)}{\Gamma\left(1+2 \alpha^{\prime} k_{1} \cdot k_{2}+2 \alpha^{\prime} k_{1} \cdot k_{4}\right)}
$$

where $k_{r}$ and $\zeta_{r}$ are momentum and polarization vectors, $\sum k_{r}=0$, and $\mathcal{N}$ is a normalization constant. The kinematic factor is defined in the usual manner

$$
K(1,2,3,4)=-16 t_{8}^{\mu_{1} \mu_{2} \mu_{3} \mu_{4} \mu_{5} \mu_{6} \mu_{7} \mu_{8}} k_{1 \mu_{1}} \zeta_{1 \mu_{2}} k_{2 \mu_{3}} \zeta_{2 \mu_{4}} k_{3 \mu_{5}} \zeta_{3 \mu_{6}} k_{4 \mu_{7}} \zeta_{4 \mu_{8}}
$$

with $t_{8}$ the well-known eighth-rank tensor, which is antisymmetric in each of the four pairs of consecutive indices and symmetric under interchange of two pairs.

Equation (2.1) can be interpreted as the amplitude for any of the processes (a), (b) or (c) that describe the scattering of closed or open strings in the background of a static Dp-brane oriented along the first $p$ spatial directions. The massless brane excitations correspond to external open strings with momentum $k_{r}$ restricted to the tangent hyperplane. Normal and tangent open-string polarizations describe, respectively, the transverse oscillations of the D-brane, and the gauge bosons living on its world-volume. Amplitudes with 
an external graviton of momentum $p_{\mu}$ and polarization $\varepsilon_{\mu \nu}$ can be obtained by the formal replacements 24,25]

$$
2 k_{1 \mu} \rightarrow p_{\mu}, 2 k_{2 \mu} \rightarrow(D \cdot p)_{\mu}, \quad \text { and } \quad \zeta_{1 \mu} \zeta_{2 \nu} \rightarrow \varepsilon_{\mu \lambda} D_{\nu}^{\lambda}
$$

where the diagonal matrix $D$ has a +1 entry for a direction tangent to the world-volume of the static Dp-brane and -1 for a normal direction. Note that the constraint $\sum k_{r}=0$ is still obeyed, even though the transverse momentum of the graviton is not necessarily conserved in the process. It can be checked that with the above identifications the lowenergy limit of these amplitudes is compatible with the Born-Infeld action (1.3) coupled to the type-II supergravity lagrangian. The comparison fixes the normalization constant

$$
\mathcal{N} \rightarrow-\frac{1}{8} T_{(p)} \alpha^{\prime 2} \prod_{\text {open }}\left(2 \pi \alpha^{\prime}\right)^{\mp 1 / 2}
$$

where the product runs over open-string external legs, and the sign depends on whether the external leg is a world-volume gauge-boson or a transverse brane oscillation. Note that our normalization of the graviton vertex operator, $G_{\mu \nu}=\eta_{\mu \nu}+2 \epsilon_{\mu \nu} e^{-i p X}$, differs from the one in reference [24].

We first consider the elastic scattering of a graviton, and expand the corresponding amplitude to subleading order in the momenta

$$
\begin{aligned}
A\left(\epsilon_{1}, p_{1} ; \epsilon_{2}, p_{2}\right) & =-\frac{1}{8} T_{(p)} \alpha^{\prime 2} K(1,2) \frac{\Gamma\left(-\alpha^{\prime} t / 4\right) \Gamma\left(\alpha^{\prime} q^{2}\right)}{\Gamma\left(1-\alpha^{\prime} t / 4+\alpha^{\prime} q^{2}\right)} \\
= & \frac{1}{2} T_{(p)} K(1,2)\left(\frac{1}{q^{2} t}+\frac{\pi^{2} \alpha^{\prime 2}}{24}+o\left(\alpha^{\prime 4}\right)\right)
\end{aligned}
$$

where $q^{2}=p_{1} \cdot D \cdot p_{1} / 2$ is the square momentum flowing along the world-volume of the Dp-brane, and $t=-2 p_{1} \cdot p_{2}$ is the momentum transfer in the transverse directions. The kinematic factor following from the identifications (2.3) reads

$$
K(1,2)=\left(2 q^{2} a_{1}+\frac{t}{2} a_{2}\right)
$$

with

$$
\begin{aligned}
a_{1} & =\operatorname{Tr}\left(\epsilon_{1} \cdot D\right) p_{1} \cdot \epsilon_{2} \cdot p_{1}-p_{1} \cdot \epsilon_{2} \cdot D \cdot \epsilon_{1} \cdot p_{2}-p_{1} \cdot \epsilon_{2} \cdot \epsilon_{1} \cdot D \cdot p_{1} \\
& -p_{1} \cdot \epsilon_{2} \cdot \epsilon_{1} \cdot D \cdot p_{1}-p_{1} \cdot \epsilon_{2} \cdot \epsilon_{1} \cdot p_{2}+q^{2} \operatorname{Tr}\left(\epsilon_{1} \cdot \epsilon_{2}\right)+\{1 \longleftrightarrow 2\}
\end{aligned}
$$




$$
\begin{aligned}
a_{2} & =\operatorname{Tr}\left(\epsilon_{1} \cdot D\right)\left(p_{1} \cdot \epsilon_{2} \cdot D \cdot p_{2}+p_{2} \cdot D \cdot \epsilon_{2} \cdot p_{1}+p_{2} \cdot D \cdot \epsilon_{2} \cdot D \cdot p_{2}\right) \\
& +p_{1} \cdot D \cdot \epsilon_{1} \cdot D \cdot \epsilon_{2} \cdot D \cdot p_{2}-p_{2} \cdot D \cdot \epsilon_{2} \cdot \epsilon_{1} \cdot D \cdot p_{1}+q^{2} \operatorname{Tr}\left(\epsilon_{1} \cdot D \cdot \epsilon_{2} \cdot D\right) \\
& -q^{2} \operatorname{Tr}\left(\epsilon_{1} \cdot \epsilon_{2}\right)-\operatorname{Tr}\left(\epsilon_{1} \cdot D\right) \operatorname{Tr}\left(\epsilon_{2} \cdot D\right)\left(q^{2}-t / 4\right)+\{1 \longleftrightarrow 2\}
\end{aligned}
$$

Note that a two-point function at tree level usually vanishes when the on-shell relations and the momentum conservation are used. In our case, since the momentum is conserved only in the directions parallel to the world-volume of the brane, the disk-level two-point function does not vanish.

The leading term in the momentum expansion of the amplitude (2.5) contains poles from the exchange of open $\left(1 / q^{2}\right)$ and closed $(1 / t)$ string states, plus contact terms, all consistent with the Dirac-Born-Infeld lagrangian and the two-derivative supergravity action. The subleading term in the amplitude, on the other hand, can be attributed entirely to the $\mathcal{O}\left(\alpha^{\prime 2}\right)$ contact interactions of two gravitons on the world-volume of the D-brane. Indeed, the trilinear vertices in the bulk as well as the graviton/ripple mixing on the world-volume are protected by supersymmetry and should not receive any string corrections. Thus there are no 1PI contributions to the subleading term of the disk amplitude. Note that corrections to the above vertices can be of course generated by field redefinitions, so that a particular choice of fields is implicitly assumed in our discussion. For bulk fields this is the standard choice in which the higher-derivative corrections to the type II supergravity lagrangian are at least (curvature) ${ }^{4}$, and are of subleading order compared to the terms considered here.

Possible $\mathcal{O}\left(\alpha^{\prime 2}\right)$ corrections to the DBI action, consistent with space-time and worldvolume reparametrization invariance, are enumerated in appendix B. They depend on various pull-backs of the ambient curvature and on the second fundamental form of the world-volume, $\Omega$. At the linearized level around flat space and a static D-brane, $\Omega$ contains an open-string excitation, and the bulk Ricci tensor $R_{\mu \nu}=R_{\mu \rho \nu}^{\rho}$ is zero. As a result (see appendix B) terms involving $\Omega$ and $R_{\mu \nu}$ do not contribute to the two-graviton amplitude (2.5). One further ambiguity is related to the Gauss-Bonnet combination

$$
\mathcal{L}_{G B}=\frac{\sqrt{g}}{32 \pi^{2}}\left(R_{\alpha \beta \gamma \delta} R^{\alpha \beta \gamma \delta}-4 \hat{R}_{\alpha \beta} \hat{R}^{\alpha \beta}+\hat{R}^{2}\right)
$$

Here and in the following $\hat{R}_{\alpha \beta}, \hat{R}_{a b}$ and $\hat{R}$ are obtained by contracting tangent indices only. The Gauss-Bonnet combination is a topological invariant in four dimensions, but it is a total derivative at quadratic order in all dimensions. Hence it does not contribute to 
the elastic scattering of a graviton off the D-brane. Modulo all these ambiguities, there exist nine remaining independent terms whose coefficients can be fixed by comparing with the string-theory amplitude (2.5).

This straightforward but tedious comparison leads to the following result for the (curvature $)^{2}$ corrections to the DBI action,

$$
\begin{aligned}
\mathcal{L}_{C P-\text { even }}^{(p)} & =c e^{-\phi} \mathcal{L}_{G B}+T_{(p)} e^{-\phi} \sqrt{g}\left[1-\frac{1}{24} \frac{\left(4 \pi^{2} \alpha^{\prime}\right)^{2}}{32 \pi^{2}} \times\right. \\
& \left.\times\left(R_{\alpha \beta \gamma \delta} R^{\alpha \beta \gamma \delta}-2 \hat{R}_{\alpha \beta} \hat{R}^{\alpha \beta}-R_{a b \alpha \beta} R^{a b \alpha \beta}+2 \hat{R}_{a b} \hat{R}^{a b}\right)+\mathcal{O}\left(\alpha^{\prime 4}\right)\right]
\end{aligned}
$$

where we included the still arbitrary coefficient, $c$, of the Gauss-Bonnet term which has dimensions of (length) ${ }^{-4}$ and which we will fix in a minute. This expression involves components of the curvature in both the tangent and the normal bundles. It is strictlyspeaking only valid for totally-geodesic embeddings of the world-volume (such that $\Omega=0$, see appendix A) in a Ricci-flat ambient space-time. We will see below how to generalize the formula to other embeddings.

The ambiguous coefficient of the Gauss-Bonnet term can be fixed by the following indirect argument. Consider type IIA theory compactified on a K3 surface, which is dual to the heterotic string on $T^{4}$ [16]. A wrapped D4-brane carries a D0-brane charge induced by the presence of the gravitational WZ term in the world-volume action (1.1) [7],

$$
-\frac{1}{48} \frac{1}{8 \pi^{2}} \int_{K 3} \operatorname{tr}(\mathrm{R} \wedge \mathrm{R})=-1
$$

Now comparison with the spectrum of the heterotic string leads to the following mass formula on the type-IIA side [27]

$$
m=T_{(4)} n_{4} V_{K 3}+T_{(0)} n_{0}
$$

with $n_{4}$ and $n_{0}$ the D4-brane and D-particle charge. The DBI action contribution to the mass is proportional to the K3 volume which does not give the required shift. However, this is given by including the $R^{2}$ term in the action. The presence of this term results in a contribution that is proportional to the Euler class of the manifold,

$$
\chi \equiv \int_{K 3} d^{4} \zeta \mathcal{L}_{G B}=\frac{1}{32 \pi^{2}} \int_{K 3} d^{4} \zeta \sqrt{g} R_{\alpha \beta \gamma \delta} R^{\alpha \beta \gamma \delta}=24 .
$$

We use here the fact that K3 is Ricci flat, and that all curvatures with normal-bundle indices are zero. Substituting in (2.10) and using the standard expressions for the tensions 
$T_{(0)}$ and $T_{(4)}$ gives the correct BPS mass formula for the type IIA theory compactified on $\mathrm{K} 3$, provided we set the coefficient $c=0$.

We will note two pieces of evidence that give partial confirmation of our result. First, consider a D-particle sitting at a point of a static curved space-manifold, such as a CalabiYau space. It can be checked that the only potential $\mathcal{O}\left(\alpha^{\prime 2}\right)$ corrections in this case could come from the contraction of two curvatures with all indices in the normal bundle. Such terms are explicitly absent in (2.10), consistently with the fact that the mass of the Dparticle should not receive any $\alpha^{\prime}$ corrections. space-time supersymmetry. Consider a $\mathcal{N}=4$ supersymmetric compactification of type II theory to four dimensions with 16 D3-branes and 64 orientifold three-planes. We will discuss this compactification in more detail in section 3. The gravitational Wess-Zumino term receives contributions both from the D3-branes and from the O3-planes, adding up altogether to 24 times the contribution of one D3-brane [18]. Supersymmetry relates these CP-odd Wess-Zumino terms to their CP-even counterparts (see, for example, [28]). If we assume that the coefficients of the CP-odd (curvature) ${ }^{2}$ terms for an O3-plane and a D3-brane are in the same proportion as their CP-even counterparts, then the coefficient of $R_{\alpha \beta \gamma \delta} R^{\alpha \beta \gamma \delta}$ agrees with the one found above. This confirms independently the choice of the Gauss-Bonnet coefficient $c=0$.

Let us consider finally the case of arbitrary world-volume embeddings, which are not totally geodesic $(\Omega \neq 0)$. We have found that the following lagrangian

$$
\begin{aligned}
\mathcal{L}_{C P-\text { even }}^{(p)} & =T_{(p)} e^{-\phi} \sqrt{g}\left[1-\frac{1}{24} \frac{\left(4 \pi^{2} \alpha^{\prime}\right)^{2}}{32 \pi^{2}}\left(\left(R_{T}\right)_{\alpha \beta \gamma \delta}\left(R_{T}\right)^{\alpha \beta \gamma \delta}-\right.\right. \\
& \left.\left.-2\left(R_{T}\right)_{\alpha \beta}\left(R_{T}\right)^{\alpha \beta}-\left(R_{N}\right)_{\alpha \beta a b}\left(R_{N}\right)^{\alpha \beta a b}+2 \bar{R}_{a b} \bar{R}^{a b}\right)+\mathcal{O}\left(\alpha^{\prime 4}\right)\right],
\end{aligned}
$$

generalizes (2.10) and reproduces correctly the two other scattering amplitudes, (b) and (c), computed in [25]. These are the amplitudes involving open strings, which correspond to geometric brane excitations (but not to world-volume gauge bosons). In the above lagrangian $R_{T}$ and $R_{N}$ are the world-volume curvature and the $S O(9-p)$ gauge field strength, which in the case of a totally geodesic embedding reduce to the pull-backs of the ambient curvature (see appendix A), and we have defined $\bar{R}_{a b} \equiv \hat{R}_{a b}+g^{\alpha \alpha^{\prime}} g^{\beta \beta^{\prime}} \Omega_{a \mid \alpha \beta} \Omega_{b \mid \alpha^{\prime} \beta^{\prime}}$. As previously, our arguments cannot fix the coefficients of terms involving the bulk Ricci tensor or the trace of the second fundamental form - both of which vanish by virtue of the

6 We thank M. Douglas for this argument. 
mass-shell conditions at linearized level. We have not checked whether comparison with the above scattering amplitudes leaves any residual ambiguities in the $\mathcal{O}\left(\alpha^{\prime 2}\right)$ disk-level action, beyond those corresponding to local redefinitions of the geometric brane coordinates $Y^{\mu}(\zeta)$.

The CP-odd (curvature) ${ }^{2}$ terms in the disk-level D-brane action derived in [9] must involve closed forms on the world-volume, in order to be invariant under gauge transformations of the $\mathcal{R} \otimes \mathcal{R}$ potentials. Hence, for non-geodesics embeddings, they must also be expressed in terms of the world-volume and $S O(9-p)$ curvatures,

$$
\mathcal{L}_{C P-o d d}^{(p)}=T_{(p)}\left(C^{(p+1)}+\frac{\pi^{2} \alpha^{\prime 2}}{24} C^{(p-3)} \wedge\left[\operatorname{tr}\left(\mathrm{R}_{T} \wedge \mathrm{R}_{T}\right)-\operatorname{tr}\left(\mathrm{R}_{N} \wedge \mathrm{R}_{N}\right)\right]\right) .
$$

We will now focus our attention on non-perturbative corrections to the D3-brane action. To this end, we will specialize for simplicity to the case where the ambient spacetime is the direct product of the worldvolume times a normal space. The terms that survive in (2.14) and (2.15) in this special case can be combined together as follows

$$
\int_{M^{(4)}} \mathcal{L}_{R^{2}}^{(3) \text { tree }}=\frac{1}{192 \pi} \int_{M^{(4)}}\left[-\tau_{2} \operatorname{tr}\left(\mathrm{R} \wedge^{*} \mathrm{R}\right)+\tau_{1} \operatorname{tr}(\mathrm{R} \wedge \mathrm{R})\right],
$$

where $\tau$ is defined in (1.4) and $\mathrm{R}$ will stand from now on for the $\mathrm{SO}(1,3)$-valued curvature two-form on the world-volume of the D3-brane.

\section{Instanton corrections to the D3-brane action}

The conjectured $S L(2, \mathbb{Z})$ invariance of type IIB theory requires that the D3-brane world-volume theory be invariant under $S L(2, \mathbb{Z})$ transformations of the supergravity backgrounds. If one keeps only the two-derivative terms in the effective action, the invariance is guaranteed by the standard Montonen-Olive duality of four-dimensional $\mathcal{N}=4$ supersymmetric Yang-Mills theory. More generally, it is also true that the sum of DBI and non-gravitational WZ terms of the D3-brane action exhibits electromagnetic duality [29,30,31]. This is consistent with $S L(2, \mathbb{Z})$ invariance in the special limit in which gravitational-curvature and covariant 'acceleration' terms can be neglected.

The $R^{2}$ terms obtained from disk amplitudes, on the other hand, do not by themselves respect $S L(2, \mathbb{Z})$ invariance. To see why, it is useful to first rescale the metric from string to the Einstein frame, $G_{\mu \nu}=e^{\phi / 2} G_{\mu \nu}^{E}$. In this frame the dilaton drops out of the Nambu-Goto action, while the parity-conserving and parity-violating $R^{2}$ terms are 
multiplied, respectively, by $e^{-\phi}$ and by $C^{(0)}$. Since $S L(2, \mathbb{Z})$ transformations do not act on the Einstein-frame metric whereas $\tau$ transforms as a modular parameter, invariance is explicitly lost. This applies both to the parity conserving terms that multiply $\tau_{2}$ and to the gravitational WZ terms that multiply $\tau_{1}$.

Our purpose in this section is to explain how D-instanton corrections to the effective D3-brane action restore invariance under $S L(2, \mathbb{Z})$. One way to understand the presence of instanton corrections is to compactify a transverse direction and then perform a Tduality tranformation that changes the D3-brane into a D4-brane. Now the D4-brane forms threshold bound states with D-particles [1], whose Euclidean trajectories can wrap around the compact dimension. Taking these Euclidean trajectories into account is equivalent in T-dual language to summing the D-instanton corrections. As it turns out the effective Wilsonian action with its instanton corrections still leads to an anomalous phase of the partition function under modular transformations. This phase cancels, however, precisely the anomalous contribution of the massless $\mathcal{N}=4$ vector multiplet living on the D-brane, provided also that the metric background satisfies an appropriate consistency condition.

\subsection{Duality between type IIA on $K 3 \times T^{2}$ and F-theory on $K 3 \times T^{4}$}

The D-instanton corrections to the $R^{2}$ terms of the D3-brane action are, in the special case of a trivial normal bundle discussed here, summarized by (1.5) . G More explicitly, the effective Wilsonian action contains a CP-even piece

$$
\int_{M^{(4)}} \mathcal{L}_{R^{2}}^{(3) \text { CP-even }}=\frac{1}{32 \pi^{2}} \int_{M^{(4)}} \log |\eta(\tau)|^{2} \operatorname{tr}\left(\mathrm{R} \wedge^{*} \mathrm{R}\right)
$$

where the logarithm of the Dedekind function has the weak-coupling expansion,

$$
\log |\eta(\tau)|^{2}=-\frac{\pi}{6} \tau_{2}-\left[q+\frac{3 q^{2}}{2}+\frac{4 q^{3}}{3} \cdots+\mathrm{cc}\right] .
$$

The first term is the coefficient of the perturbative disk diagram contribution, and the series of powers of $q=e^{2 \pi i \tau}$ are the D-instanton corrections. Likewise, the CP-odd piece of the effective Wilsonian action reads

$$
\int_{M^{(4)}} \mathcal{L}_{R^{2}}^{(3) C P-o d d}=-\frac{i}{32 \pi^{2}} \int_{M^{(4)}} \log \frac{\eta(\tau)}{\eta(\bar{\tau})} \operatorname{tr}(\mathrm{R} \wedge \mathrm{R}) .
$$

7 A non-trivial normal bundle poses some extra difficulties which we will comment on briefly later. 
where the logarithm may be expanded as

$$
\log \frac{\eta(\tau)}{\eta(\bar{\tau})}=\frac{i \pi}{6} \tau_{1}-\left[q+\frac{3 q^{2}}{2}+\frac{4 q^{3}}{3}+\cdots-\mathrm{cc}\right] .
$$

The first term, which again arises from the perturbative disk diagram, coincides with the $C^{(0)}$ term in (2.15) (for $p=3$ and when the normal bundle is trivial) whereas the series of powers of $q$ stand again for the D-instanton corrections. The derivative with respect to $\tau$ of this expression can be written in terms of the imaginary part of the second Eisenstein form, which is holomorphic but not modular covariant.

One way of arriving at the above action, outlined in reference [18], can be rephrased in terms of the duality between F-theory compactified on $K 3 \times T^{4}$ and type-IIA theory compactified on $K 3 \times T^{2}$, which is also dual to heterotic theory on $T^{6}$. The exact expression for the $R^{2}$ terms in the type IIA theory can be derived from a one-loop calculation [19, 20] and is a function of the torus Kähler modulus $T$. On the F-theory side, on the other hand, these terms come from the sum of the actions on $24(p, q)$ seven-branes compactified to four dimensions on $T^{4}$. A maximum of eighteen of these may be seven-branes with the same values of $p$ and $q$, but fortunately the $R^{2}$ terms of interest are the same for all types of seven-branes. Furthermore, a T-duality in the four $T^{4}$ directions maps these $R^{2}$ terms for a $(p, q)$ seven-brane to the corresponding terms for a D3-brane. This chain of dualities therefore predicts that the $R^{2}$ terms in the D3-brane action are exactly $1 / 24$ th of the corresponding type-IIA loop amplitude, if one identifies $\tau$ in the former with the Kähler modulus $T$ in the latter. Comparing with the exact type-IIA expression [19,20] leads then to the conjectured action (1.5) on an individual D3-brane.

Let us explain this argument in some more detail. In the case of a D7-brane, the disk-level action contains the terms

$$
\int_{M^{(8)}} \mathcal{L}_{R^{2}}^{(7)}=\frac{1}{\lambda_{s}^{B}} \frac{1}{192 \pi} \frac{1}{\left(4 \pi^{2} \alpha^{\prime}\right)^{2}} \int_{M^{(8)}}\left(-e^{-\phi} \operatorname{tr}\left(\mathrm{R} \wedge^{*} \mathrm{R}\right)+C^{(4)} \wedge \operatorname{tr}(\mathrm{R} \wedge \mathrm{R})\right) .
$$

Switching from the string to the ten-dimensional Einstein frame eliminates the dilaton dependence of this action. Since both the Einstein-frame metric and the four-form $C^{(4)}$ are inert under $S L(2, \mathbb{Z})$ transformations, we can conclude that $(3.5)$ is $S L(2, \mathbb{Z})$ invariant and must thus describe the $R^{2}$ terms in the world-volume action of any $(p, q)$ seven-brane. After compactification on $T^{4}$ the above seven-brane action reduces to the leading, disk-level term of the D3-brane action (1.5) provided we identify the modular parameter

$$
\tau=\frac{\left(4 \pi^{2} \alpha^{\prime}\right)^{-2}}{\lambda_{s}^{B}}\left(\int_{T^{4}} C^{(4)}+i v_{E}^{(4)}\right)
$$


where $v_{E}^{(4)}$ is the volume of the four-torus in the ten-dimensional Einstein frame. It can be checked that this identification is the one dictated by the T-duality transformation that maps the seven-branes to D3-branes.

¿From the seven-brane point of view the instanton corrections arise from Euclidean trajectories of D3-branes wrapped around the compact $T^{4}$ and the quantity $q=e^{2 \pi i \tau}$ is the semi-classical measure corresponding to a singly-wrapped trajectory. 8 It is worth noting that in the eight-dimensional compactification of F-theory both the dilaton and the conformal factor of the metric of the $\sigma$-model that is induced on the seven-branes vary as functions of the transverse two-dimensional space. This variation however precisely cancels out (see for instance [37]) when one considers the Einstein metric, which is consistent with the fact that the modulus $v_{E}^{(4)}$ should not depend on the precise locations of the sevenbranes.

It is important to check that the instanton corrections to the Wess Zumino action do not affect the anomaly inflow argument of [8]. For example, consider the configuration in which a D3-brane intersects a D7-brane on a line, along which chiral fermions propagate. The consequent gravitational anomalies in the intersection domain are supposed to be cancelled by anomalous inflow from the branes. The anomalous variation of the WZ action of the D3-brane under space-time reparametrizations depends only on the source term in the anomalous Bianchi identity for the $\mathcal{R} \otimes \mathcal{R}$ one-form field strength. But this is not affected by the non-perturbative D-instanton corrections because the semi-classical measure $q$ has trivial monodromy around the D7-brane,

$$
\int_{S^{1}} d \log \frac{\eta(\tau)}{\eta(\bar{\tau})}=\frac{i \pi}{6 \lambda_{s}^{B}} \int_{S^{1}} d C^{(0)}=\frac{i \pi}{6}
$$

where $S^{1}$ is a circle around the D7-brane. The anomalous variation in the intersection domain therefore comes entirely from the disk-level part of the D3-brane WZ action. A similar argument should ensure that instanton effects in the D7-brane do not affect the inflow argument.

As we will explain in the concluding remarks, a chain of reasoning analogous to the one described in this subsection also requires the presence of nonperturbative $R^{4}$ contributions to the seven-brane action, coming from localized D-instantons.

8 See [32,33, 34, 35, 36] for discussions of such extended D-brane instantons in other contexts. 


\subsection{Modular anomalies}

The elements of $S L(2, \mathbb{Z})$ are generated by the transformations $T$, which acts as a shift $\tau \rightarrow \tau+1$, and $S$, which acts as the inversion $\tau \rightarrow-1 / \tau$. The corresponding transformations of the logarithm of the Dedekind function are

$$
\log \eta(\tau+1)=\log \eta(\tau)+i \frac{\pi}{12}, \quad \log \eta(-1 / \tau)=\log \eta(\tau)-i \frac{\pi}{4}+\frac{1}{2} \log \tau
$$

As a result, the effective Wilsonian action (1.5) is not by itself $S L(2, \mathbb{Z})$ invariant. This situation is familiar from other related examples [38,39,40,41]. The $\tau$-dependent variation of the Wilsonian action should cancel the anomalous contributions of the massless modes, which are the gauge boson and its fermionic partners in the abelian $\mathcal{N}=4$ vector multiplet that describes the low-energy dynamics of the D-brane. The constant part of the variation (3.8) on the other hand, leads to a net anomalous variation equal to the integral of a topological density - the first Pontryagin class of the manifold wrapped by the worldvolume of the D3-brane. This is an integer multiple of $2 \pi$ in consistent backgrounds, for which the first Pontryagin class is an integer multiple of 24 .

To understand the modular anomaly of the massless D3-brane modes, we need to consider the action for $\mathcal{N}=4$ supergravity coupled to the $\mathcal{N}=4$ Maxwell theory 44, 43. Both the Maxwell field and its fermionic partners contribute anomalous variations of the partition function. One way of analyzing the anomaly of the fermions is to start with a gauge-invariant description of the IIB theory in which there are three scalar fields, $\tau, \bar{\tau}$ and $\phi$, that parameterize the group manifold of $S L(2, \mathbb{R})$. These package into a zweibein, $V_{i}^{a}$, which is an $S L(2, \mathbb{R})$ matrix

$$
V_{i}^{a}=\frac{1}{\sqrt{\tau_{2}}}\left(\begin{array}{cc}
\tau_{2} \cos \phi+\tau_{1} \sin \phi & -\tau_{2} \sin \phi+\tau_{1} \cos \phi \\
\sin \phi & \cos \phi
\end{array}\right) .
$$

The global $S L(2, \mathbb{R})$ acts by matrix multiplication on $V$ from the left while the local $U(1)$ acts from the right. The $U(1)$ gauge potential is the composite field

$$
a_{\mu}=\partial_{\mu} \phi-\frac{\partial_{\mu} \tau_{1}}{2 \tau_{2}}
$$

The field $\phi$ transforms as

$$
\phi(x) \rightarrow \phi(x)+\Sigma(x)
$$

under the local $U(1)$ (where $0 \leq \Sigma \leq 2 \pi$ is the gauge parameter) and by

$$
\phi \rightarrow \phi-\frac{i}{2} \log \left(\frac{c \tau+d}{c \bar{\tau}+d}\right)
$$


under the $S L(2, \mathbb{R})$ transformation which acts on the field $\tau$ in the usual manner,

$$
\tau \rightarrow A \tau=\frac{a \tau+b}{c \tau+d} \quad \text { where } \quad A=\left(\begin{array}{ll}
a & b \\
c & d
\end{array}\right)
$$

Notice that $a_{\mu}$ is invariant under $S L(2, \mathbb{R})$.

This local $U(1)$ also acts as a phase rotation on the four Weyl fermions whose charge is $-1 / 2$. On the other hand the fermions are inert under $S L(2, \mathbb{R})$. In the absence of anomalies the field $\phi$ can be eliminated by fixing the gauge, and then the remaining scalars $\tau$ and $\bar{\tau}$ parameterize the coset $S L(2, \mathbb{R}) / U(1)$ where the $U(1)$ is identified with a compact $S O(2) \equiv U(1)$ subgroup of $S L(2, \mathbb{R})$. At first this appears to be impossible, even in the absence of D-branes, because of an apparent anomalous coupling of the $U(1)$ current to four gravitons and the $U(1)$ field strength. This arises from an anomalous hexagon diagram with circulating chiral gravitini and dilatini. However, the anomalous $U(1)$ transformation of the fermion determinant can be cancelled by a local counterterm proportional to $\int \phi \mathrm{F} \wedge \operatorname{trR}^{4}$ [4]. The modular anomaly in ten dimensions arises because of the transformation of $\phi$ under $S L(2, \mathbb{Z})$ which leads to an anomalous variation of this counterterm.

A similar phenomenon occurs on the world-volume of the D3-brane where a triangle anomaly arises from the one-loop diagram coupling the divergence of the $U(1)$ current to two gravitons. The world-volume gauginos which have a chiral coupling to the $U(1)$ are circulating inside the loop. The anomalous phase of the partition function is given by the standard expression

$$
\delta S_{\text {gauginos }}=-4 \times \frac{1}{2} \int_{M^{(4)}} \Sigma(x) I_{1 / 2}(R)=\frac{1}{96 \pi^{2}} \int_{M^{(4)}} \Sigma(x) \operatorname{tr}(\mathrm{R} \wedge \mathrm{R})
$$

where $I_{1 / 2}(R)$ is the Dirac index and the overall coefficient follows from the $U(1)$ charge of the four Weyl fermions. This $U(1)$ anomaly can be cancelled by the addition of a counterterm in the world-volume action

$$
\int_{M^{(4)}} \mathcal{L}_{\text {counter }}^{(3)}=-\frac{1}{96 \pi^{2}} \int_{M^{(4)}} \phi \operatorname{tr}(\mathrm{R} \wedge \mathrm{R}) .
$$

Since $\phi$ is an angular variable this expression is unambiguous only if the first Pontryagin class is an integer multiple of 12 - a milder restriction on consistent backgrounds than the one that we will encounter shortly. Although (3.15) cancels the local $U(1)$ anomaly it violates the symmetry of the action under $S L(2, \mathbb{Z})$ transformations since $\phi$ transforms as 
in (3.12). The $T$ transformation is nonanomalous but under an $S$ transformation (3.15) transforms by

$$
\delta S_{\text {gauginos }}=\frac{i}{192 \pi^{2}} \int_{M^{(4)}} \log \left(\frac{\tau}{\bar{\tau}}\right) \operatorname{tr}(\mathrm{R} \wedge \mathrm{R}),
$$

which is therefore the fermionic contribution to the modular anomaly. Put differently, we can use the gauge symmetry to eliminate the auxiliary field $(\phi=0)$, but then an $S L(2, \mathbb{Z})$ transformation must be accompanied by a compensating $U(1)$-gauge transformation, under which the fermionic determinants would have exactly the same anomalous variation as above.

This fermionic contribution only cancels part of the $\tau$-dependence in the modular transformation of (3.1) and (3.3). An additional contribution to the modular anomaly arises from the action of $S L(2, \mathbb{Z})$ on the world-volume Maxwell field strength 45,46]. The Maxwell field is inert under the local $U(1)$, so one must calculate directly the anomaly in the current of the $S L(2, \mathbb{R})$ symmetry which is a good global symmetry of the lowenergy equations of motion. It should be possible to calculate this anomaly as a triangle diagram with two external gravitons and one insertion of the $S L(2, \mathbb{R})$ current. Here we will instead use directly the results of [45,46] for the anomalous transformation of the Maxwell field partition function. As with the fermion determinant, the $T$ transformation is nonanomalous but the $S$ transformation leads to the anomalous phase,

$$
\delta S_{\text {Maxwell }}=\frac{1}{32 \pi^{2}} \operatorname{Re} \int_{M^{(4)}} \log \tau\left[-\frac{1}{2} \epsilon_{\alpha \beta \gamma \delta} \mathrm{R}^{\alpha \beta} \wedge \mathrm{R}^{\gamma \delta}+\frac{2 i}{3} \operatorname{tr}(\mathrm{R} \wedge \mathrm{R})\right] .
$$

This combines with the contribution of the fermion determinant, (3.16), to cancel the $\tau$-dependent part of the variation of the CP-odd term (3.3), which is the contribution of the massive string modes. Likewise, the anomalous phase of the Maxwell partition function cancels the variation of the CP-even term (3.1) for Ricci-flat backgrounds for which $\frac{1}{2} \epsilon_{\alpha \beta \gamma \delta} \mathrm{R}^{\alpha \beta} \wedge \mathrm{R}^{\gamma \delta}=\operatorname{tr}\left(\mathrm{R} \wedge{ }^{*} \mathrm{R}\right)$. Recall that our D3-brane action was only obtained modulo terms involving the Ricci tensor of the bulk.

The modular transformation (3.8) has also a $\tau$-independent part which gives rise to an anomalous phase equal to a constant multiple of $\int \operatorname{tr}(\mathrm{R} \wedge \mathrm{R})$. For consistency this must be an integer multiple of $2 \pi$. This condition is fulfilled by consistent compactifications for which the first Pontryagin class is an integer multiple of 48 ,

$$
\frac{1}{48} p_{1}(R)=-\frac{1}{48} \frac{1}{8 \pi^{2}} \int \operatorname{tr}(\mathrm{R} \wedge \mathrm{R}) \in \mathbb{Z} .
$$

This condition is fullfilled in particular in the special case of a K3 surface. 
It is also interesting to understand how the modular anomaly cancels in the F-theory background of section 3.1, or equivalently the heterotic theory compactified on $T^{6}$. We have already argued that the net contribution to the $\tau$-dependent part of the anomaly from massive string modes is equal to 24 times that of a single D3-brane, in accordance with the result of [19,20]. This should cancel the $\tau$-dependent part of the modular anomaly coming from the $\mathcal{N}=4$ supergravity coupled to $U(1)^{22}$ super-Yang-Mills theory, which is the effective theory at a generic point of moduli space. To see how this works out precisely, one must use the fact that the $U(1)$ charges of the gravitini, dilatini and gaugini are, respectively, $\frac{1}{2},-\frac{3}{2}$ and $-\frac{1}{2}$, and that the dilatini have opposite chirality compared to the other fermionic fields 42,43. Furthermore, the expression of the Dirac index for a spin $\frac{3}{2}$ field is 47

$$
I_{3 / 2}(R)=I_{1 / 2}(R)\left(D-1-\operatorname{tr}\left(e^{i \mathrm{R} / 2 \pi}-\mathbf{1}\right)\right)=-21 I_{1 / 2}(R)+\cdots
$$

where in the second equality we consider only the four-form relevant for $D=4$ spacetime dimensions. Finally the chirality of the graviphotons is opposite to that of the gauge bosons. Putting all this together one finds the following contributions to the modular anomaly, in units in which the contribution of an entire $\mathcal{N}=4$ vector multiplet is one,

$$
\underbrace{22 \times \frac{2}{3}}_{\text {gauge bosons }}-\underbrace{22 \times\left(-\frac{1}{2}\right) \times \frac{4}{6}}_{\text {gaugini }}-\underbrace{6 \times \frac{2}{3}}_{\text {graviphotons }}+\underbrace{\left(-\frac{3}{2}\right) \times \frac{4}{6}}_{\text {dilatini }}+\underbrace{21 \times \frac{1}{2} \times \frac{4}{6}}_{\text {gravitini }} .
$$

The total is indeed 24, consistently with the absence of anomalies as well as with the heterotic/type IIA/F-theory duality conjectures.

\section{M-theory interpretation}

Another strategy for obtaining the nonperturbative $R^{2}$ contributions is to make use of the dualities that relate string theory to M-theory. For example, the bulk $R^{4}$ terms in the effective action of ten-dimensional type II string theory can be obtained from the one-loop Feynman diagrams that contribute to four-graviton scattering in elevendimensional supergravity compactified on $T^{2}$ [23]. The quanta circulating around the loop are interpreted as D-particles in the type IIA and D-instantons in the type IIB descriptions of the theory. The correspondence requires the identification of the complex IIB coupling $\tau=\tau_{1}+i \tau_{2}$ with the complex structure of the two-torus in M-theory,

$$
\tau \longleftrightarrow U=\frac{R_{9}}{R_{11}} e^{i \theta}
$$


where $\theta$ is the angle of the torus whose cycles have length $2 \pi R_{9}$ and $2 \pi R_{11}$. This identification follows from the standard relations connecting type-IIA theory and M-theory:

$$
R_{11}=l_{s} \lambda_{s}^{A}, \quad l_{P}=\left(\frac{2 \kappa_{(11)}^{2}}{(2 \pi)^{8}}\right)^{1 / 9}=l_{s}\left(\lambda_{s}^{A}\right)^{1 / 3}
$$

as well as the T-duality transformation relating the IIA and IIB descriptions:

$$
r^{B}=l_{s}^{2} / r^{A}, \quad \lambda_{s}^{B}=\lambda_{s}^{A} \sqrt{\frac{r^{B}}{r^{A}}} .
$$

Here $r^{A}=R_{9} \sin \theta$ and $r^{B}$ are the radii of the ninth dimension in the IIA and IIB respectively descriptions, $l_{s}=\sqrt{\alpha^{\prime}}$ is the string length scale and $l_{P}$ the eleven-dimensional Planck length. The expectation value of the $\mathcal{R} \otimes \mathcal{R}$ scalar is $C^{(0)}=\cot \theta$. The tendimensional type IIB string theory is recovered in the limit of vanishing $T^{2}$ volume, $V^{(2)}=4 \pi^{2} R_{9} R_{11} \sin \theta \rightarrow 0$, with fixed complex structure.

As we will now show the $R^{2}$ term in the CP-even part of the D3-brane and D4brane actions can also be obtained by an extension of this argument. We consider Mtheory in the presence of an M5-brane that is wrapped around the cycles of a two-torus. This is interpreted as a D4-brane in the type IIA description and a D3-brane in the type IIB interpretation. We will consider a one-loop diagram with two external gravitons scattering in the world-volume of the M5-brane. The circulating quanta with non-vanishing momentum in the eleventh direction are now interpreted as bound states of D-particles with the D4-brane. In the type IIB description they correspond to the D-instantons in the D3-brane whose contributions have been resummed in expression (1.5).

This one-loop effect can be in principle computed as the sum of Feynman diagrams with the component fields of the chiral $(2,0)$ tensor multiplet of the M5-brane circulating in the loop. A more efficient method for obtaining this expression is to make use of the manifestly supersymmetric first-quantized light-cone gauge formalism of [23] in which the amplitude is described as a trace over the states of the superparticle circulating around the loop and coupled to two external gravitons. This method, inspired by the stringtheory calculation, will be sufficient to find the CP-even part of the action. The elevendimensional coordinates are labelled $x^{0}, \ldots x^{9}, x^{11}$ and the light-cone gauge is defined so that $X^{+}(t)=\left(X^{0}(t)+X^{1}(t)\right) / \sqrt{2}=p^{+} t+x^{+}$. The two-torus will be chosen to be in the $x^{9}, x^{11}$ directions while the M5-brane will be oriented in the $x^{0}, x^{1}, x^{2}, x^{3}, x^{9}, x^{11}$ 
directions. The light-cone gauge vertex operator of a graviton with transverse momentum $p_{i}(i=2, \ldots, 9,11)$ and polarization $\epsilon_{i j}$ is 48

$$
V(\epsilon, p)=2 \pi\left(\frac{1}{32} \mathcal{S} \gamma^{i l} \mathcal{S} p_{l} \mathcal{S} \gamma^{j m} \mathcal{S} p_{m}+\cdots\right) \epsilon_{i j} e^{i p \cdot X}
$$

(where it is also necessary to set $p^{+}=0$ as usual in defining light-cone gauge vertex operators). The real $S O(9)$ spinor $\mathcal{S}^{a}(a=1, \ldots, 16)$ is the $\gamma^{+} \gamma^{-}$projection of a covariant $S O(10,1)$ spinor and it satisfies the anticommutation relations $\left\{S^{a}, S^{b}\right\}=\delta^{a b}$. We have only indicated here the term in $V(\epsilon, p)$ which is quartic in the spinor coordinates, since other terms do not contribute to the amplitude of interest. We have also fixed the overall normalization so as to make contact with the string-theory amplitude of section 2 .

In evaluating the loop amplitude, we can restrict ourselves to the special kinematic setup in which the polarizations of the two external gravitons, as well as their momenta, are in the directions $x^{2}, x^{3}$ which are the world-volume directions transverse to the light-cone and $T^{2}$ directions. This will be sufficient if we are not interested in the normal bundle. More generally, it is sufficient that the polarizations and momenta have no components in the eleventh dimension. The expression for the loop amplitude is :

$$
A\left(\epsilon_{1}, p_{1} ; \epsilon_{2}, p_{2}\right)=\frac{1}{2 V^{(2)}} \sum_{l_{9}, l_{11}} \int \frac{d^{4} k}{(2 \pi)^{4}} \operatorname{Tr}_{\mathcal{S}} \prod_{r=1,2} \int_{0}^{\infty} d t_{r} V\left(\epsilon_{r}, p_{r}\right) e^{-\pi\left(G^{I J} l_{I} l_{J}+k_{r}^{2}\right) t_{r}}
$$

where the trace is over the fermionic modes and $r=1,2$ labels the two graviton vertices. The four-dimensional continuous momenta in the legs of the loop are denoted $k_{r}=k+\sum_{s=1, r} p_{r}$ and $G^{I J} l_{I} l_{J}$ is the square of the Kaluza-Klein momentum in the compact directions, $x^{9}$ and $x^{11}$. This special configuration of momenta and polarizations is sufficient to determine the parity-conserving part of the amplitude. However, a more general configuration would be necessary to extract the parity-violating piece which is proportional to the world-volume form and has vector indices associated with all four world-volume directions.

We will first discuss the trace over the fermionic modes, $\mathcal{S}$. The M5-brane breaks half of the thirty-two supersymmetries, preserving those of definite chirality $\gamma^{0} \gamma^{1} \gamma^{2} \gamma^{3} \gamma^{9} \gamma^{11}$. Decomposing the $S O(9)$ spinor $\mathcal{S}$ into two eight-dimensional Majorana-Weyl $S O(8)$ spinors $S_{R}$ and $S_{L}$, this relation translates into the usual relation between right and left moving spinors in the type II theories in the presence of a D-brane,

$$
S_{L}=\gamma^{2} \gamma^{3} \gamma^{9} S_{R} \equiv \gamma^{(3)} S_{R},
$$


Substituting this decomposition into the trace, the kinematic factor takes the form

$$
K(1,2)=\frac{1}{16} \operatorname{Tr}_{S_{R}} \prod_{r=1,2} \epsilon_{r, i j} p_{r, l} p_{r, m}\left(S_{R} \gamma^{i l} S_{R}\right)\left(S_{R} \gamma^{(3)} \gamma^{j m} \gamma^{(3)} S_{R}\right) .
$$

Now one can use the relation $\gamma^{(3)} \gamma^{j m} \gamma^{(3)}=\gamma^{j^{\prime} m^{\prime}} D_{j^{\prime}}{ }^{j} D_{m^{\prime}}{ }^{m}$, with $D$ the matrix introduced in section 2 , and the well known fact that the trace over eight $S_{R}$ 's is proportional to the $t_{8}$ tensor. The final result agrees precisely with the kinematic factor $K(1,2)$ given in eqs. (2.6-8).

Next, we shall evaluate the integrals over the momenta and over the two proper times $t_{r}$. Since we are only interested in the four-derivative terms, we can set to zero the external momenta in the integrand. Then, letting $t \equiv t_{1}+t_{2}$, and integrating over the loop momentum $k$ and over the difference $\left(t_{1}-t_{2}\right) / 2$ we find

$$
A\left(\epsilon_{1}, p_{1} ; \epsilon_{2}, p_{2}\right)=\frac{K(1,2)}{128 \pi^{2} V^{(2)}} \int_{0}^{\infty} \frac{d t}{t} \sum_{l_{9}, l_{11}} e^{-\pi t\left|l_{9}-l_{11} \tau\right|^{2} / v^{(2)} \tau_{2}}
$$

We have here used the explicit form for the square of the Kaluza-Klein momentum

$$
G^{I J} l_{I} l_{J}=\frac{4 \pi^{2}}{V^{(2)} \tau_{2}}\left|l_{9}-l_{11} \tau\right|^{2}
$$

where $\tau=U$ is the complex structure of the torus, and we have absorbed a factor of $4 \pi^{2}$ by a rescaling of $t$. Poisson resumming over $l_{9}$ leads to

$$
A\left(\epsilon_{1}, p_{1} ; \epsilon_{2}, p_{2}\right)=\frac{K(1,2)}{128 \pi^{2}}\left(\frac{\tau_{2}}{V^{(2)}}\right)^{1 / 2} \int_{0}^{\infty} \frac{d t}{t^{3 / 2}} \sum_{w_{9}, l_{11}} e^{-\pi \tau_{2}\left(w_{9}^{2} v^{(2)} / t+l_{11}^{2} t / v^{(2)}\right)-2 \pi i \tau_{1} l_{11} w_{9}}
$$

which is the starting point for analyzing the various contributions.

In the analysis that follows, we will see that the Wilsonian action in ten non-compact dimensions corresponds to the $l_{11} \neq 0$ contributions in the above sum, that is to non-zero momentum along the hidden eleventh dimension of string theory. On the other hand, the contributions with $l_{11}=0$ and $w_{9} \neq 0$ will reproduce the one-loop open-string amplitude, which is saturated by massless modes in the ten-dimensional IIA $\left(R_{9} \rightarrow \infty\right)$ or IIB $\left(R_{9} \rightarrow\right.$ $0)$ theory. We may further separate the $l_{11} \neq 0$ sum into contributions with $w_{9} \neq 0$ and 
$w_{9}=0$. The terms with $w_{9} \neq 0$ give a

$$
\begin{aligned}
\mathcal{A}^{\text {non pert }}=\frac{1}{128 \pi^{2}}\left(\frac{\tau_{2}}{V^{(2)}}\right)^{1 / 2} & \int_{0}^{\infty} \frac{d t}{t^{3 / 2}} \sum_{w_{9} \neq 0, l_{11} \neq 0} e^{-\pi \tau_{2}\left(v^{(2)} w_{9}^{2} / t+l_{11}^{2} t / v^{(2)}\right)-2 \pi i l_{11} w_{9} \tau_{1}} \\
& =\frac{\tau_{2}^{1 / 2}}{64 \pi^{2} V^{(2)}} \sum_{w_{9} \neq 0, l_{11} \neq 0}\left|\frac{l_{11}}{w_{9}}\right|^{1 / 2} K_{1 / 2}\left(2 \pi\left|w_{9} l_{11}\right| \tau_{2}\right) e^{-2 \pi i w_{9} l_{11} \tau_{1}} \\
& =\frac{1}{64 \pi^{2} V^{(2)}} \sum_{N=1}^{\infty} \sum_{n \mid N} \frac{1}{n}\left(e^{2 \pi i N \tau}+\text { c.c. }\right)
\end{aligned}
$$

This reproduces precisely the non-perturbative D-instanton sum in the expansion (3.2) of the CP-even part of the effective action, and gives an independent confirmation of our non-perturbative conjecture.

The terms with $w_{9}=0$ and any value of $l_{11}$ (including the term with $l_{11}=0$ ) are quadratically ultraviolet divergent. The total divergence may be isolated by converting the sum over the Kaluza-Klein charge, $l_{11}$, to a sum over winding number in the eleventh dimension, $w_{11}$, by the Poisson resummation,

$$
\begin{aligned}
\mathcal{A}^{\text {tree }}=\frac{1}{128 \pi^{2}}\left(\frac{\tau_{2}}{V^{(2)}}\right)^{1 / 2} \int_{0}^{\infty} \frac{d t}{t^{3 / 2}} \sum_{l_{11}} e^{-\pi \tau_{2} l_{11}^{2} t / v^{(2)}} & =\frac{1}{128 \pi^{2}} \int_{0}^{\infty} \frac{d t}{t^{2}} \sum_{w_{11}} e^{-\pi v^{(2)} w_{11}^{2} / \tau_{2} t}= \\
& =\frac{1}{64 \pi^{3}} \zeta(2) \frac{\tau_{2}}{V^{(2)}}+\frac{1}{128 \pi^{2}} \int_{0}^{\infty} \frac{d t}{t^{2}}
\end{aligned}
$$

The finite piece reproduces the tree-level part of the effective D-brane action (noting $\zeta(2)=\pi^{2} / 6$ and that the M-theory amplitude must be multiplied by the size of the hidden dimension, $2 \pi R_{11}$ in order to make the comparison with the string amplitude).

The divergence is now isolated entirely in the zero winding number term, $w_{9}=$ $w_{11}=0$. In any microscopic definition of M-theory this divergence would be regularized and replaced by a finite value. However, we can argue that its regularized value must be zero by an elementary use of duality. This follows from the fact that in the decompactified theory $\left(R_{9}, R_{11} \rightarrow \infty\right)$ the divergent term corresponds to a $R^{2}$ term in the effective fivebrane action, proportional to $1 / l_{p}^{2}$ which is the only scale in the theory. Compactifying one large dimension $\left(x^{11}\right)$ would then give a $R^{2}$ term proportional to

$$
R_{11} / l_{p}^{2}=\left(\lambda_{s}^{A}\right)^{2 / 3} / l_{s}
$$

Since a fractional power of the string coupling constant has no sensible interpretation in the IIA theory, we conclude that the divergent piece in (4.12) must be discarded. 
The remaining terms in the double sum (4.8) that have not been accounted for are the terms with $l_{11}=0$ and $w_{9} \neq 0$. These add up to

$$
\mathcal{A}^{\text {one loop }}=\frac{1}{128 \pi^{2}}\left(\frac{\tau_{2}}{V^{(2)}}\right)^{1 / 2} \int_{0}^{\infty} \frac{d t}{t^{3 / 2}} \sum_{w_{9} \neq 0} e^{-\pi v^{(2)} \tau_{2} w_{9}^{2} / t}
$$

corresponding precisely to the one-loop contributions of the BPS open-string modes living on the D-brane. These BPS states consist of the massless vector multiplet on the D3-brane, as well as of its winding-mode descendants when some transverse dimensions are compact. Notice that the $w_{9}=0$ term in the annulus diagram must be subtracted in computing the 1PI action, since it corresponds to a (potential) tree-exchange of a supergraviton [49]. The sum (4.14) has a logarithmic divergence which is responsible for the CP-even one-loop modular anomaly of section 3 .

The weakly-coupled type IIA theory is obtained in the limit $R_{11} \rightarrow 0, R_{9} \rightarrow \infty$, in which the M5-brane reduces to the D4-brane. In this case, the instanton terms vanish and the amplitude reduces to the perturbative terms. The weakly-coupled type IIB theory on the other hand is obtained in the limit of vanishing volume for the two-torus, $V^{(2)} \rightarrow 0$ with $\tau=U$ held fixed. The $N$ th term in the non-perturbative sum (4.11) comes from the sector of $\mathrm{N}$ D-instantons (and anti D-instantons) confined to the world-volume of the brane. The measure $\sum_{n \mid N} 1 /|n|$ can be identified with the bulk term in the Witten index of the T-dual quantum mechanics problem of $N$ D-particles propagating in the world-volume of the D4-brane. This is analogous to the case of the ten-dimensional D-instanton where the measure is $\sum_{n \mid N} 1 / n^{2}$ [50]. The extra factor of $1 /|n|$ comes from the fact that there are an unequal number of bosonic and fermionic moduli in the ten-dimensional case whereas these numbers are equal in the D4-brane. The arguments of [51,52,50] suggest that the boundary contribution to the Witten index is $1-\sum_{n \mid N} 1 /|n|$ so that the total Witten index is equal to one. This agrees with the expectation that there should be threshold bound states of this system.

In principle, we should also be able reproduce the parity violating $\mathrm{R} \wedge \mathrm{R}$ term in (1.5) by considering a loop correction in the M5-brane, but we have not done this. Nevertheless, from (1.5) and using the usual T-duality relations between the IIB and IIA theories it also follows that the instanton terms disappear in the D4-brane. In that case, the gravitational contribution to the WZ term in the D4-brane action is simply proportional to $C^{(1)} \wedge \operatorname{tr}(\mathrm{R} \wedge$ $\mathrm{R})$, as in [7,8,9]. 


\section{Comments and discussion}

We have given various arguments for establishing the $R^{2}$ terms that are relevant to the gravitational interactions of p-branes in string and M-theory. These terms are the first nontrivial corrections in the low energy expansion of the world-volume action and are relevant for a p-brane in a curved background. Even at this low order we have left several questions unanswered. For example, we determined the complete action for geodesic and non geodesic world-volume embeddings at tree level (a disk world-sheet), including contributions from nontrivial normal bundle. However, our discussion of the nonperturbative effects was restricted to situations in which the normal bundle is trivial.

The discussion of nonperturbative effects in section 4 was incomplete in two respects. Firstly, we again limited our discussion to situations in which the normal bundle is trivial. Secondly, it was limited to the parity-conserving part of the action. These restrictions arose, in part, from our use of a light-cone formalism which cannot be applied in any obvious manner to the most general kinematic configurations. Since the M5-brane plays a central and subtle role in the circle of dualities it would be interesting to understand in detail how the $\mathrm{R} \wedge \mathrm{R}$ terms in the D3-brane can be deduced from a loop calculation in the M5-brane. The restriction to configurations with trivial normal bundle was more than a technical convenience. There is a well-known problem [53,54,55] with chiral anomalies that arises when embedding the M5-brane in M theory. After toroidal compactification and T duality to the type IIB theory the normal bundle is associated with the chiral $S O(6) \sim S U(4) \mathrm{R}$ symmetry of the D3-brane embedded in ten dimensions. The possible mixed anomalies in this symmetry and in $S L(2, \mathbb{Z})$ will require special restrictions on terms in the worldvolume action that involve the normal bundle curvature. These should be analogous to the terms involving the tangent bundle curvature that we found are necessary for the absence of modular anomalies.

Another issue that we have not addressed in any detail is the structure of higher curvature terms in seven-branes. In section 3 we saw how the $R^{2}$ terms on a compactified seven-brane are related by T-duality to those of the D3-brane. However, we know that there are also $R^{4}$ terms in the seven-brane world-volume action. According to (1.1) the disklevel CP-odd WZ term in the world-volume action of a D7-brane is of the form $C^{(0)} Y_{8}(R)$, where $Y_{8}(R)$ denotes an eight-form that is the linear combination of $\operatorname{tr}(\mathrm{R} \wedge \mathrm{R} \wedge \mathrm{R} \wedge \mathrm{R})$ and $(\operatorname{tr}(\mathrm{R} \wedge \mathrm{R}))^{2}$ that follows from (1.2). Similarly, considerations of scattering on the seven-brane world-volume will lead to CP-even $R^{4}$ terms. Arguments similar to those 
given in this paper imply the presence of nonperturbative contributions to these $R^{4}$ terms. The necessity for such contributions can be seen in particular by considering the F-theory background consisting of $24(p, q)$ seven-branes, which is dual to the heterotic or type-I string compactified to eight dimensions on $T^{2}$. The known structure of the $R^{4}$, as well as the $R^{2} F^{2}$ and $F^{4}$ terms in this background [33, 35, 34, 56, 57, 58, 59] implies that in the type-I language the quartic action of both orientifold seven-planes and D7-branes should receive non-trivial D-instanton corrections. Although we will not discuss the details here, it should again be possible to motivate the form of these nonperturbative $R^{4}$ terms by the absence of modular anomalies. Furthermore, as with the $R^{2}$ terms in the D3-brane, the M-theory origin of such terms should emerge from duality relations between type-I' string theory on $T^{2}$ or F-theory on K3 and eleven-dimensional M-theory compactified on $T^{2} \times I$ with $I$ the finite-length interval of the Horava-Witten configuration [60]. The D-instanton contributions on the string theory side should presumably arise from a one-loop calculation of four graviton scattering in the above compactification of M-theory.

Finally, we have not addressed interesting issues concerning the compatibility of the curvature terms in the D3-brane world-volume action and the correspondence between type IIB superstrings in $A d S_{5} \times S^{5}$ and $\mathcal{N}=4$ supersymmetric Yang-Mills theory. One obvious issue is the consistency of the higher-order curvature terms in the world-volume action of a test D3-brane probe parallel to the $A d S_{5}$ boundary. These curvature terms are a priori nonzero since the Riemann curvature is nonzero in this background. However, there are surely other world-volume terms that we have not addressed, which include powers of the self-dual fifth-rank field strength, $F_{5}=d C^{(4)}$, which is also nonzero in this background. It would be interesting to check whether these corrections are consistent with the statement that supersymmetry protects the energy per unit volume of a stack of $\mathrm{N}$ parallel D3branes. Another interesting question is how the D-instanton terms in the D3-brane action contributions are interpreted in terms of the contributions of Yang-Mills instantons.

\section{Aknowledgements}

We are grateful to E. Cremmer, M. Douglas, K. Förger, M. Gaberdiel, G. Gibbons, R. Minasian, M. Petropoulos, B. Pioline and S. Silva for useful discussions, and to A. Tseytlin for a critical reading of the manuscript. 


\section{Appendix A. The geometry of submanifolds}

We will here collect some standard facts on the geometry of submanifolds which can be found in standard texts, such as [61,62]. The embedding of the D-brane worlvolume in the ambient spacetime is described by the ten coordinate functions $Y^{\mu}\left(\zeta^{\alpha}\right)$, with $\mu=$ $0, \cdots, 9$ a space-time index and $\alpha=0, \cdots p$ a world-volume index. The tangent vectors $\partial_{\alpha} Y^{\mu}$ define a local frame for the tangent bundle. We may also define an orthonormal frame for the normal bundle, $\xi_{a}^{\mu}$ with $a=p+1, \cdots 9$, such that

$$
\xi_{a}^{\mu} \xi_{b}^{\nu} G_{\mu \nu}=\delta_{a b} \quad \text { and } \quad \xi_{a}^{\mu} \partial_{\alpha} Y^{\nu} G_{\mu \nu}=0
$$

Both the $\partial_{\alpha} Y^{\mu}$ and the $\xi_{a}^{\mu}$ transform as vectors under target-space reparametrizations. Furthermore the former are vectors of world-volume reparametrizations, while the latter transform as vectors under local rotations of the normal bundle.

With the help of these local frames we can pull back any tensor from the target space-time onto the tangent and/or normal bundles by contraction. The only non-trivial pull-back of the ambient metric is the induced world-volume metric

$$
g_{\alpha \beta}=G_{\mu \nu} \partial_{\alpha} Y^{\mu} \partial_{\beta} Y^{\nu} .
$$

The Riemann tensor, on the other hand, can be pulled back in many different ways. Using its symmetry properties, and the fact that the sum over a cyclic permutation of three indices is zero, we can write down the following six independent pull-backs,

$$
R_{\alpha \beta \gamma \delta}, R_{\alpha \beta \gamma a}, R_{\alpha \beta a b}, R_{\alpha\{a b\} \beta}, R_{\alpha a b c} \text { and } R_{a b c d},
$$

where the curly brackets denote the symmetric combination of the two normal (and hence also the two tangent) indices. These pulled-back Riemann curvatures transform as tensors of world-volume reparametrizations and normal-frame rotations, but are scalars under reparametrizations of the target space. We may furthermore contract tangent indices with $g_{\alpha \beta}$ and normal indices with $\delta_{a b}$. Since on the D-brane world-volume the ambient metric can be decomposed as

$$
G^{\mu \nu}=\partial_{\alpha} Y^{\mu} \partial_{\beta} Y^{\nu} g^{\alpha \beta}+\xi_{a}^{\mu} \xi_{b}^{\nu} \delta^{a b}
$$

the contraction of spacetime indices before being pulled back does not give rise to any new tensors. 
Covariant derivatives can be defined with the target-space connection $\Gamma_{\nu \rho}^{\mu}$, the affine world-volume connection $\left(\Gamma_{T}\right)_{\beta \gamma}^{\alpha}$ constructed out of the induced metric, and the composite $\mathrm{SO}(9-\mathrm{p})$ gauge field

$$
\omega_{\alpha}^{a b}=\xi^{\mu,[a}\left(G_{\mu \nu} \partial_{\alpha}+G_{\mu \sigma} \Gamma_{\nu \rho}^{\sigma} \partial_{\alpha} Y^{\rho}\right) \xi^{\nu, b]}
$$

which is defined implicitly by requiring that the normal frame be covariantly constant. One important tensor is the covariant derivative of the tangent frame, also known as the 'second fundamental form'

$$
\Omega_{\alpha \beta}^{\mu}=\Omega_{\beta \alpha}^{\mu}=\partial_{\alpha} \partial_{\beta} Y^{\mu}-\left(\Gamma_{T}\right)_{\alpha \beta}^{\gamma} \partial_{\gamma} Y^{\mu}+\Gamma_{\nu \rho}^{\mu} \partial_{\alpha} Y^{\nu} \partial_{\beta} Y^{\rho}
$$

It is a symmetric world-volume tensor and a vector of the ambient space-time. Using the fact that $g_{\alpha \beta}$ is covariantly-constant one can show that the tangent-space projection of $\Omega$ vanishes, $\Omega_{\alpha \beta}^{\gamma}=0$. With no loss of information we may thus project it on the normal bundle

$$
\Omega_{\alpha \beta}^{a} \equiv \Omega_{\alpha \beta}^{\mu} \xi^{\nu, a} G_{\mu \nu}
$$

In the special case of a point-particle trajectory, $\Omega_{\tau \tau}^{\mu}$ is the covariant acceleration which is everywhere normal to the world line. A submanifold with zero second-fundamental form is called auto-parallel or totally geodesic.

The central equations in the theory of submanifolds are the Gauss-Codazzi equations. They relate the world-volume curvature $R_{T}$, constructed out of the affine connection $\Gamma_{T}$, and the field strength $R_{N}$ of the $S O(9-p)$ gauge connection $\omega$, to pull-backs of the space-time Riemann tensor plus combinations of the second fundamental form,

$$
\left(R_{T}\right)_{\alpha \beta \gamma \delta}=R_{\alpha \beta \gamma \delta}+\delta_{a b}\left(\Omega_{\alpha \gamma}^{a} \Omega_{\beta \delta}^{b}-\Omega_{\alpha \delta}^{a} \Omega_{\beta \gamma}^{b}\right)
$$

and

$$
\left(R_{N}\right)_{\alpha \beta}^{a b}=-R_{\alpha \beta}^{a b}+g^{\gamma \delta}\left(\Omega_{\alpha \gamma}^{a} \Omega_{\beta \delta}^{b}-\Omega_{\alpha \gamma}^{b} \Omega_{\beta \delta}^{a}\right)
$$

Notice that if the ambient space-time is flat these world-volume curvatures can be expressed entirely in terms of the second fundamental form $\Omega$. Conversely, if the world-volume is a totally geodesic manifold the curvature forms of the induced connections coincide with the pull-backs of the ambient curvature. 


\section{Appendix B. The systematics of (curvature $)^{2}$ terms}

We will here discuss the systematics of possible $\mathcal{O}\left(\alpha^{2}\right)$ terms which can appear as part of the D-brane actions. Invariance under reparametrizations of the world-volume and of the ambient space, as well as under local rotations of the normal frame, dictate that possible terms are formed by full contractions of covariant tensors. At linearized level around flat space and a static D-brane background the curvature tensor is proportional to a closed-string (graviton) excitation, and the second fundamental form is proportional to an open-string (geometric brane) excitation. We assume that all vertices that do not contain at least two gravitons, four open strings, or one graviton and two open strings, are protected by supersymmetry and receive no higher-order corrections. Thus the terms that will interest us are of the type $R^{2}, R \Omega^{2}$ and $\Omega^{4}$ - all of which are $\mathcal{O}\left(\alpha^{\prime 2}\right)$.

As discussed in the main text, our arguments cannot determine any terms that vanish by virtue of the lowest-order equations of motion. These equations impose the vanishing of the bulk Ricci tensor and of the trace of the second fundamental form,

$$
R_{\nu \mu \rho}^{\mu}=0 \quad \text { and } \quad \Omega^{\mu} \equiv g^{\alpha \beta} \Omega_{\alpha \beta}^{\mu}=0 .
$$

Since both $R_{\mu \nu}$ and $\Omega^{\mu}$ will vanish at linearized level by virtue of the mass-shell conditions, such terms do not contribute to the amplitudes of interest.

We will first consider the case $\Omega_{\alpha \beta}^{\mu}=0$, corresponding to totally-geodesic embeddings. Since $\Omega$ contains an open-string excitation at the linearized level, this restriction is appropriate when comparing with the two-graviton amplitude (2.5). One set of allowed terms are the squares of the six curvature pull-backs (A.3),

$$
\begin{aligned}
& R_{\alpha \beta \gamma \delta} R^{\alpha \beta \gamma \delta}, \quad R_{\alpha \beta \gamma a} R^{\alpha \beta \gamma a}, \quad R_{\alpha \beta a b} R^{\alpha \beta a b}, \\
& R_{\alpha\{a b\} \beta} R^{\alpha\{a b\} \beta}, \quad R_{\alpha a b c} R^{\alpha a b c}, \quad R_{a b c d} R^{a b c d} .
\end{aligned}
$$

A second set of invariants can be constructed by squaring the (partially or fully) contracted pulled-back curvatures. The partial contractions of the six Riemann curvatures in (A.3) give rise to six different two-index tensors. However, the vanishing of the bulk Ricci tensor imposes three independent relations among them. We will choose as the three independent two-index tensors those obtained by contracting tangent indices, and will denote them by $\hat{R}_{\alpha \beta}, \hat{R}_{a \alpha}$, and $\hat{R}_{a b}$ (the hat is there to remind us that these tensors are not the pull-backs 
of the bulk Ricci tensor). Further contraction of the remaining indices gives rise to a single independent scalar $\hat{R}$. In total there are therefore four new possible quadratic terms,

$$
\hat{R}_{\alpha \beta} \hat{R}^{\alpha \beta}, \quad \hat{R}_{\alpha a} \hat{R}^{\alpha a}, \quad \hat{R}_{a b} \hat{R}^{a b}, \quad \hat{R}^{2} .
$$

Comparison with the two-graviton amplitude fixes the coefficients of all the terms in (B.2) and (B.3), modulo one residual ambiguity corresponding to the Gauss-Bonnet combination.

Let us turn now to invariants constructed with the second fundamental form $\Omega$. Recalling that $\Omega$ is traceless, we find four terms of the type $\Omega^{4}$,

$$
\begin{array}{ll}
\left(\Omega_{\alpha \beta} \cdot \Omega^{\alpha \beta}\right)\left(\Omega_{\gamma \delta} \cdot \Omega^{\gamma \delta}\right), & \left(\Omega_{\alpha \gamma} \cdot \Omega^{\alpha \delta}\right)\left(\Omega^{\beta \gamma} \cdot \Omega_{\beta \delta}\right), \\
\left(\Omega_{\alpha \beta} \cdot \Omega_{\gamma \delta}\right)\left(\Omega^{\alpha \beta} \cdot \Omega^{\gamma \delta}\right), & \left(\Omega_{\alpha \beta} \cdot \Omega_{\gamma \delta}\right)\left(\Omega^{\alpha \gamma} \cdot \Omega^{\beta \delta}\right),
\end{array}
$$

where we have suppressed in an obvious notation the normal index of $\Omega$. Likewise, there are six invariants of the type $\Omega^{2} R$,

$$
\begin{array}{llc}
R^{\alpha \beta \gamma \delta}\left(\Omega_{\alpha \gamma} \cdot \Omega_{\beta \delta}\right), & R_{a b}^{\alpha \beta} \Omega_{\alpha \gamma}^{a} \Omega_{\beta}^{b \gamma}, & R_{\{a b\}}^{\alpha \beta} \Omega_{\alpha \gamma}^{a} \Omega_{\beta}^{b \gamma}, \\
\hat{R}^{\alpha \beta}\left(\Omega_{\alpha \gamma} \cdot \Omega_{\beta}^{\gamma}\right), & \hat{R}_{a b} \Omega_{\alpha \beta}^{a} \Omega^{b \alpha \beta}, & \hat{R}\left(\Omega_{\alpha \beta} \cdot \Omega^{\alpha \beta}\right) .
\end{array}
$$

One can use the Gauss-Codazzi equations to rewrite some of the above invariants in terms of the world-volume curvatures $R_{T}$ and $R_{N}$. There are four possible contractions of these curvatures,

$$
\left(R_{T}\right)_{\alpha \beta \gamma \delta}\left(R_{T}\right)^{\alpha \beta \gamma \delta},\left(R_{T}\right)_{\alpha \beta}\left(R_{T}\right)^{\alpha \beta},\left(R_{T}\right)^{2} \text { and }\left(R_{N}\right)_{\alpha \beta}^{a b}\left(R_{N}\right)_{a b}^{\alpha \beta} .
$$

Four additional invariants are the cross terms involving one world-volume curvature and the corresponding pull-back tensor. All these terms are linear combinations of the invariants (B.4) and (B.5). As discussed in the main text we have found an action that reproduces correctly the three scattering amplitudes of section 2 . We did not however analyze systematically the most general combination of the above invariants for $\Omega \neq 0$.

We have also excluded from our considerations terms of the type $D^{2} R, \Omega D^{2} \Omega, \Omega^{2} D \Omega$ or $\Omega D R$, other than those obtained after an integration by parts from one of the invariants discussed before. Such terms, if present, would have given $\mathcal{O}\left(\alpha^{2}\right)$ corrections to the one-point function of the graviton, the propagator of an open string, the vertex of three open strings, or to the mixing of an open with a closed string - all of which are presumably protected by supersymmetry for some field choice. As a check of this argument we have verified explicitly that $D^{2} R$ and $\Omega D R$ terms would, if present, make unacceptable contributions to the three string amplitudes discussed in section 2 . 


\section{References}

[1] J. Polchinski, TASI Lectures on D-Branes, hep-th/9611050.

[2] C.Bachas, Lectures on D-branes, hep-th/9806199.

[3] J. Polchinski, Dirichlet-Branes and Ramond-Ramond Charges, hep-th/9510017; Phys. Rev. Lett. 75 (1995) 4724.

[4] C.G. Callan, C. Lovelace, C.R. Nappi and S.A. Yost, Loop Corrections to Superstring Equations of Motion, Nucl. Phys. 308 (1988) 221.

[5] M. Li, Boundary States of D-branes and Dy-Strings, hep-th/9510161; Nucl. Phys. B460 (1996) 351.

[6] M. Douglas, Branes within branes, hep-th/9512077.

[7] M. Bershadsky, V. Sadov and C. Vafa, D-branes and topological field theories, hepth/9511222; Nucl. Phys. B462 (1996) 420.

[8] M.B. Green, J.A. Harvey and G. Moore, I-brane inflow and anomalous couplings on D-branes, hep-th/9605033; Class. Quant. Grav. 14 (1997) 47.

[9] Y-K. E. Cheung and Z. Yin, Anomalies, branes and currents, hep-th/9710206; Nucl. Phys. B517 (1998) 69.

[10] B. Craps and F. Roose, Anomalous D-brane and orientifold couplings from the boundary state, hep-th/9808074; Phys.Lett. B445 (1998) 150-159; (Non-)Anomalous Dbrane and O-plane couplings: the normal bundle, hep-th/9812149.

[11] B. Stefanski, Gravitational Couplings of D-branes and O-planes, hep-th/9812088.

[12] J.F. Morales, C.A. Scrucca and M. Serone, Anomalous couplings for D-branes and O-planes, hep-th/9812071.

[13] E.S. Fradkin and A. Tseytlin, Non-linear Electrodynamics from Quantized Strings, Phys. Lett. B163 (1985) 123.

[14] R.G. Leigh, Dirac-Born-Infeld action from Dirichlet sigma model, Mod. Phys. Lett. A4 (1989) 2767.

[15] C. Bachas, D-brane Dynamics, hep-th/9511043; Phys. Lett. B374 (1996) 37.

[16] C.M. Hull and P. K. Townsend, Unity of Superstring Dualities, hep-th/9410167; Nucl. Phys. B438 (1995) 109-137

[17] J.H. Schwarz, An SL(2,Z) Multiplet of Type IIB Superstrings, hep-th/9508143; Phys. Lett. B360 (1995) 13-18; Erratum-ibid. B364 (1995) 252.

[18] K. Dasgupta, D.P. Jatkar and S. Mukhi, Gravitational couplings and $Z_{2}$ orientifolds, hep-th/9707224: Nucl. Phys. B523 (1998) 465.

[19] J. Harvey and G. Moore, Fivebrane Instantons and $R^{2}$ couplings in $\mathcal{N}=4$ String Theory, hep-th/9610237; Phys. Rev. D57 (1998) 2323-2328.

[20] A. Gregori, E. Kiritsis, C. Kounnas, N. A. Obers, P. M. Petropoulos and B. Pioline, $R^{2}$ Corrections and Non-perturbative Dualities of $\mathcal{N}=4$ String ground states, hepth/9708062; Nucl. Phys. B510 (1998) 423-476. 
[21] J.H. Schwarz, The Power of M-Theory, hep-th/9510086; Phys. Lett. B367 (1996) 97-103.

[22] P.S. Aspinwall, Some relationships between dualities in string theory, hep-th/9508154 Nucl. Phys. Proc. Suppl. 46 (1996) 30-38.

[23] M. B. Green, M. Gutperle and P. Vanhove, One loop in eleven dimensions, hepth/9706175; Phys. Lett. B409 (1997) 177-184.

[24] M.R. Garousi and R.C. Myers, Superstring scattering from D-branes, hep-th/9603194; Nucl. Phys. B475 (1996) 193-224; World-volume interactions on D-branes, hepth/9809100; Nucl.Phys. B542 (1999) 73-88.

[25] A. Hashimoto, I. R. Klebanov, Decay of Excited D-branes, hep-th/9604065; Phys. Lett. B381 (1996) 437-445; Scattering of Strings from D-branes, hep-th/9611214; Nucl. Phys. Proc. Suppl. 55B (1997) 118-133.

[26] M. Green, J. Schwarz and E. Witten, Superstring theory, Cambridge U. Press (1987).

[27] S. Ramgoolam and D. Waldram, Zero-Branes on a Compact Orbifold, hep-th/9805191; J.High Energy Phys. 9807 (1998) 009.

[28] S. Theisen, Fourth order supergravity, Nucl. Phys. B263 (1986) 687; Addendum-ibid. B269 (1986) 744.

[29] G.W. Gibbons and Rasheed, SL(2,R) Invariance of Non-Linear Electrodynamics Coupled to An Axion and a Dilaton, hep-th/9509141; Phys. Lett. B365 (1996) 46-50.

[30] A. Tseytlin, Self-duality of Born-Infeld action and Dirichlet 3-brane of type IIB superstring theory, hep-th/9602064; Nucl. Phys. B469 (1996) 51-67.

[31] M.B. Green and M. Gutperle, Comments on D3-branes, hep-th/9602077; Phys. Lett. B377 (1996) 28-35.

[32] H. Ooguri and C. Vafa, Summing up D-Instantons, hep-th/9608079; Phys. Rev. Lett. 77 (1996) 3296-3298.

[33] C. Bachas, C. Fabre, E. Kiritsis, N. A. Obers and P. Vanhove, Heterotic / type I duality and D-brane instantons, hep-th/9707126; Nucl. Phys. B509 (1998) 33-52.

[34] C. Bachas, Heterotic versus Type I, hep-th/9710102; Nucl. Phys. Proc. Suppl. 68 (1998) 348-354.

[35] E. Kiritsis and N. Obers, Heterotic/Type-I Duality in D $<10$ Dimensions, Threshold Corrections and D-Instantons, hep-th/9709058; J. High Energy Phys. 9710 (1997) 004.

[36] I. Antoniadis, B. Pioline and T. R. Taylor, Calculable $e^{-1 / \lambda}$ Effects, hep-th/9707222; Nucl. Phys. B512 (1998) 61-78.

[37] I. Antoniadis and C. Bachas, Branes and the Gauge Hierarchy, hep-th/9812093; Phys. Lett. B450 (1999) 83.

[38] J. P. Derendinger, S. Ferrara, C. Kounnas and F. Zwirner, On loop corrections to string effective field theories: field-dependent gauge couplings and $\sigma$-model anomalies, Nucl. Phys. B372 (1992) 145. 
[39] G.L. Cardoso, D. Lust, and B.A. Ovrut, Moduli dependent non-holomorphic contributions of massive states to gravitational couplings and $C^{2}$ terms in $Z(N)$ orbifold compactification, hep-th/9410056; Nucl. Phys. B436 (1995) 65.

[40] V. Kaplunovsky and J. Louis, On gauge couplings in string theory, hep-th/9502077; Nucl. Phys. B444 (1995) 191.

[41] V. Kaplunovsky and J. Louis, Field dependent gauge couplings in locally supersymmetric effective quantum field theories, hep-th/9402005; Nucl. Phys. B422 (1994) 57.

[42] M. de Roo, Matter coupling in $\mathcal{N}=4$ Supergravity, Nucl. Phys. B255 (1985) 515.

[43] E. Bergshoeff, I. Koh and E. Sezgin, Coupling of Yang Mills to $\mathcal{N}=4, d=4$ Supergravity, Phys. Lett. 155B (1985) 71.

[44] M. Gaberdiel and M. Green, An $S L(2, \mathbb{Z})$ anomaly in IIB supergravity and its F-theory interpretation, hep-th/9810153; J. High Energy Phys. 9811 (1998) 026.

[45] C. Vafa and E. Witten, A Strong Coupling Test of S-Duality, hep-th/9408074; Nucl. Phys. B431 (1994) 3-77.

[46] E. Witten, On S-duality in Abelian gauge Theory, hep-th/9505186; Mod. Phys. Lett. A10 (1995) 2153; E. Verlinde, Global Aspects of Electric-Magnetic Duality, hepth/9506011; Nucl. Phys. B455 (1995) 211.

[47] L. Alvarez-Gaumé and P. Ginsparg, The structure of gauge and gravitational anomalies, Ann. Phys. 161 (1985) 423; Erratum-ibid. 171 (1986) 233.

[48] M.B. Green, M. Gutperle and H. Kwon, Sixteen Fermion and Related Terms in Mtheory on $T^{2}$, hep-th/9710151; Phys. Lett. B421 (1998) 149.

[49] C. Bachas and E. Kiritsis, $F^{4}$ Terms in $\mathcal{N}=4$ String Vacua, hep-th/9611205; Nucl.Phys.Proc.Suppl. 55B (1997) 194-199.

[50] M.B. Green and M. Gutperle, D-particle bound states and the D-instanton measure, hep-th/9711107; J. High Energy Phys. 9801 (1998) 005.

[51] P. Yi, Witten Index and Threshold Bound States of D-Branes, hep-th/9704098; Nucl. Phys. B505 (1997) 307-318.

[52] S. Sethi and M. Stern, D-Brane Bound States Redux, hep-th/9705046; Commun. Math. Phys. 194 (1998) 675-705.

[53] E. Witten, Five-Brane Effective Action In M-Theory, hep-th/9610234; J.Geom.Phys. 22 (1997) 103-133.

[54] L. Bonora, C. S. Chu and M. Rinaldi, Perturbative Anomalies of the M-5-brane, hepth/9710063; JHEP 9712 (1997) 007, Anomalies and Locality in Field Theories and $M$ theory, hep-th/9712205.

[55] D. Freed, J. A. Harvey, R. Minasian and G. Moore, Gravitational Anomaly Cancellation for M-Theory Fivebranes, hep-th/9803205 ; Adv.Theor.Math.Phys. 2 (1998) 601-618.

[56] W. Lerche and S. Stieberger, Prepotential, Mirror Map and F-Theory on K3, hepth/9804176. 
[57] K. Foerger and S. Stieberger, Higher Derivative Couplings and Heterotic-Type I Duality in Eight Dimensions, hep-th/9901020.

[58] W. Lerche, S. Stieberger and N. P. Warner, Prepotentials from Symmetric Products, hep-th/9901162.

[59] W. Lerche, S. Stieberger and N. P. Warner, Quartic Gauge Couplings from K3 Geometry, hep-th/9811228.

[60] P. Horava and E. Witten, Heterotic and Type I String Dynamics from Eleven Dimensions, hep-th/9510209; Nucl. Phys. B460 (1996) 506; Eleven-Dimensional Supergravity on a Manifold with Boundary, hep-th/9603142; Nucl. Phys. B475 (1996) 94.

[61] L.P. Eisenhart, Riemannian geometry, Princeton U. Press, 1926.

[62] S. Kobayashi and K. Nomizu, Foundations of Differential Geometry, J. Wiley \& Sons, New York 1969, vol.2. 